\title{
ESP Algorithms Study Cornering Light Vehicle Lateral Deviation and Yaw Joint Control
}

\author{
Liu Hongtao
}

\begin{abstract}
Automotive Engineering institute, Jiangxi University of Technology, Nanchang 330098, China
\end{abstract}
Keywords: Light Vehicle Lateral Deviation; Yaw Joint Control; ESP; BP network

\begin{abstract}
The electronic stability program can conduct real-time monitoring of driver's steering intention and vehicle status, identification of electronic control unit and determine the vehicle's steering characteristics and trends in the loss of steering stability through vehicle-mounted online sensor system. It can also prevent the vehicle appear oversteer and excessive understeer and other dangerous situations exceeding the driver's operating range through comprehensively regulating engine torque and wheel braking, to achieve ideal trajectory runing, while improving the vehicle's directional stability and handling characteristics. Based on referring to the foreign advanced ESP technology research experience, aiming at ESP electronic control unit hardware solution as well as ESP lateral deviation and yaw joint control algorithm based on fuzzy neural network controller, this paper has obtained the anticipated research results.
\end{abstract}

\section{Introduction}

When the vehicle runs in extreme steering conditions, the tire has non-linear lateral deviation characteristics; if the lateral force exceeds the adhesion limit of the tires and the ground, it will cause excessive steering or excessive understeer, resulting in poor vehicle handling stability and even loss of steering stability [4]. Studies have shown that: Automotive installation ABS (Anti-locking Brake System, Anti-Lock Braking System) and TCS (Traction Control System) is useless for the conditions aforesaid. When the steering stability control has been powerless; ESP can realize controlling the vehicle handling stability state through interventions of wheel braking torque and engine output torque, to ensure the vehicle run with correct trajectory and prevent oversteer and excessive understeer that exceed the capacity of driver's steering control. In Europe, the annual loss due to traffic accidents is amounted to 5 billion Euros, accounting for $0.5 \%$ of total EU GDP, and this figure will double by 2010. The research of Germany Audi (Audi) company shows that: As vehicle speed increasing, the number of vehicle skidding accidents has increased dramatically, the speed between $80 \sim 100 \mathrm{~km} / \mathrm{h}$, and $40 \%$ personal injury accidents are relating to skidding, whereas when the vehicle speed reaches $160 \mathrm{~km} / \mathrm{h}$ or more, almost all of the accidents are caused by skidding. ESP can increase vehicle stability and reduce the risk of skidding. A research report from Swedish National Road Administration in May 2013 shows that [8]: the accident rate of the vehicles that are equipped with ESP running on wet and snowy road can be decreased by $20 \%$ and $40 \%$. Daimler Chrysler's statistics show that: the accident rate was reduced from $22 \%$ in 1998 to $12 \%$ in 2004 since 2000 when ESP was configured as a standard configuration of Benz passenger cars; a corresponding ESP research topic of application driver simulator of the United States IOWA (Iowa NY) University found that: the vehicle equipped with ESP system could reduce more than $88 \%$ out of control probability under 
emergency steering situation than the vehicles without ESP system; at the same bad condition, the former driver casualties probability was $24.5 \%$ lower than the latter. In 2003, 53\% Volkswagen vehicles were equipped with ESP, while the statistics is that: the accident rate was reduced by $20 \%$ than 2003 after overall assembly of ESP control system.

With respect to the ABS and TCS, ESP system can obviously improve the handling stability for extreme steering conditions. Now, ASB, TCS and ESP have been integrated into a new generation of ESP.

\section{ESP working principle}

ESP system basic configuration includes: wheel speed sensor, longitudinal acceleration sensor, lateral acceleration sensor, yaw angle rate sensor, steering angle sensor, integrated HCU with brake master cylinder pressure sensor (Hydraulic control unit, hydraulic control unit), engine torque, control units, ESP electronic control unit and so on.

ESP sensor is the wheel speed sensor, steering wheel angle sensor, yaw angle rate sensor, acceleration sensor and master cylinder pressure sensor that is now widely used,.

1)Wheel speed sensor: ESP electronic control unit calculates wheel angular velocity based on the wheel speed sensor square wave signal. Currently the adopted Hall wheel speed sensor has advantages of good low-speed response characteristics, signal magnitude regardless of the speed advantages.

2)Steering wheel angle sensor: steering wheel angle sensor is used for measuring the steering wheel angle that reflects the driver's intention of steering; the absolute steering wheel angle sensor that outputs direct information such as steering angle and rotational direction is generally to measure steering angle based on the principle of grating. The handling stability information of described vehicle nominal state can be achieved through processing steering wheel angle, estimating vehicle speed and estimating sideslip.

3) Yaw angle rate sensor and acceleration sensor: yaw angle rate sensor is used for measuring the rotation angular velocity bypassing the vertical axis of the centroid, which provides basis for the electronic control unit determining whether the vehicle is excessive or too much understeer tendency and the occurrence degree; acceleration sensor is used to measure the vehicle longitudinal and lateral acceleration, together with yaw angle rate sensor to achieve the vehicle driving state real-time monitoring.

4) Brake master cylinder pressure sensor: brake master cylinder pressure sensor detects the master cylinder pressure information, which is used to estimate the wheel brake cylinder pressure. ESP electronic control unit estimates the wheel cylinder pressure in accordance with the pressure sensor measured values of the brake master cylinder and wheel cylinder brake pressure control state, and thus realizes coordinated control of longitudinal force and lateral force on the wheel.

Hydraulic control

Hydraulic control unit is an important executive body of ESP. The hydraulic control units used in the car include wheel cylinder solenoid valve, isolation valve, hydraulic pump and motor, brake master cylinder pressure sensor and solenoid valve driving board and so on.

In normal braking, the brake pressure regulating units are not energized, and the brake fluid flows into brake wheel cylinder through isolation valve 1 and booster valve. Brake pressure regulating device does not affect the normal braking. ESP system in working: if the brake wheel cylinder brake pressure needs to be increased, intake valve 1 is energized to open and isolation valve 1 is energized 
and shut off, electric pump 1 running, brake fluid entering into the brake wheel cylinder, brake pressure increased; if the brake wheel cylinder pressure needs to be maintained, suction valve 1 shall be shut off, to prevent pressure increasing, pressure valve energized and shut off, reducing valve 1 shut off, brake wheel cylinder disconnected with the outside line, the brake wheel cylinder pressure remaining constant;

if the brake wheel cylinder brake pressure needs to be reduced, booster valve and reducing valve are energized, the intake valve 1 and the booster valve shut off, reducing and isolation valve 1 opened, brake fluid will go into the low pressure accumulator firstly to ease hydraulic impact caused by high pressure brake fluid, electric pump 1 working, the brake fluid in brake wheel cylinder will flow back to the master cylinder, brake wheel cylinder pressure will be reduced.

ESP engine torque control unit

ESP engine torque control system includes an electronic throttle or engine torque adjustment unit based on CAN bus communication. In addition to adjusting the engine torque, transmission gear regulation can also be adjsted.

ESP electronic control unit

ESP electronic control unit is the core of vehicle stability control system, to process the information from various sensors and driving the actuators; at the same time, it is the carrier of control logic, brain of the system. The electronic control units generally comprise two microprocessors, two processors to exchange information with each other through an internal bus; in addition to the microprocessor, electronic control unit also comprises peripheral circuits such as power management module, sensor signal input module, hydraulic actuator driver module, each indicator interface and CAN bus communication interface.

ESP controller calculates the actual value and nominal value of vehicle handling stability state, based on the signal collected by sensors that reflects the driver's intention and vehicle status, comparing the actual value and nominal value, to determine the operating status of the vehicle and driver's intention deviation, and then applies yaw moment by adjusting the wheel brake.

ESP determines whether to adjust brake by comparing the driver's operation intention and actual running state of the vehicle. When the vehicle is oversteer, the front outer wheels will be applied e braking to generate yawing moment which is opposite with the direction of oversteer movement(clockwise in the figure), thereby suppressing excessive car steering movement; when the car is too inadequate understeering, the rear inside wheel will be applied braking for through to generate the same direction of movement of cars understeer yaw moment (counterclockwise in the figure), suppress excessive understeering movement. ESP is an active safety device to improve car stability in extreme steering conditions, which applies necessary intervention timely for automotive brake system and engine based on car viewer making full judgment on car working conditions and running state.

\section{Results}

ESP hardware-in-loop (HIL) test bench takes a light-duty vehicle as the target model, based on Matlab / xPC research and development. ESP HIL test bench includes: ESP sensor system and testing sensor, real-time platform (embedded signal processing system), actuator and actuator drive board.

(1) ESP test sensors and sensor systems. ESP system comprises wheel speed sensors, yaw rate, longitudinal acceleration, lateral acceleration, steering wheel angle and son on; test sensor includes sensors for testing brake pressure. 
a) ESP sensor system - steering angle sensor selects incremental optical encoder. It uses photoelectric conversion principle to output three groups of square wave pulse A, B and Z phase; A, $\mathrm{B}$ pulse phase difference is 90 degrees, which can easily determine the direction of rotation, and Z-phase is one pulse per revolution for reference point positioning; and other ESP sensor signal value can be calculated based on the simulation model using the I / O board simulation issuing;

b) Pressure sensor is PA-21PRO from a Swiss company called Keller, with measuring range of 0 $20 \mathrm{MPa}$, and output is $4 \sim 20 \mathrm{~mA}$ current signal. Through installing $250 \Omega$ pure resistor in series, the current signal is translated into standard voltage signal of $1 \sim 5 \mathrm{~V}$. There are totally six pressure sensors, in which pressure sensor 1 and 2 used to measure line pressure at the exit of brake master cylinder; pressure sensor 3,4,5,6 are used to measure wheel cylinder pressure.

(2) Real-time platform (embedded signal processing system), real-time platform includes three computers: one for the host, the other two for the target machines.

a) Host function: compile the vehicle model and control algorithm into real-time code running in target machine;

b) Target function: the real-time code operating the vehicle model and control algorithm,.

c) Host and two goal-machine communication: wireless network mode;

d) Signal processing system: include $\mathrm{I} / \mathrm{O}$ signal acquisition and output modules. I / O boards use Advantech PCL-818L, Advantech PCL-726 and NI PCI-6601 three models; Advantech PCL-818L is used for inputting longitudinal acceleration, lateral acceleration, yaw rate, ESP on (off), steering

Based on the above test platform, integrate the ESP control algorithm and dynamics model, for typical working conditions test, in order to verify the ESP cornering yaw joint control algorithm is effective.

By ESP hardware in the loop test curve analysis of the three typical operating conditions of variable attachment homogeneous pavement steering wheel rotation angle step-input, centralis attachment homogeneous pavement emergency lane changing, centralis attachment homogeneous pavement snake type lane, we can draw the following conclusions:

1) For the cars without ESP control, the actual value of vehicle yaw rate far exceeds yaw rate nominal value, the absolute value of centroid sideslip angle is greater than $0.15 \mathrm{rad}$, and more than the maximum sideslip angle absolute value set up by ESP HIL test bench. When sideslip angle exceeds $0.15 \mathrm{rad}$, exceeding the capacity of the steering on the car generating compensation yaw moment to correct vehicle handling stability state, leading to a sharp increase in car centroid sideslip angle and yaw rate, the driver will lose control of car side way movement ability, and the car will lose steering stability.

2) The same trend off-line with simulation results: the cars with ESP lateral deviation and yaw joint control, the difference between the actual yaw angular velocity value and its nominal value is controlled within a certain range; the centroid sideslip angle absolute value is limited within 0.15rad, which is under the control of the driver; the brake pressure will be adjusted in real time according to the car's yaw state, generating adequate compensation yaw moment to suppres car oversteer and excessive understeer phenomenon; centroid lateral acceleration is limited in the range of ground attachment permission, so the car is always in a stable traveling state; the control system hardware, especially considering the brake hydraulic actuator response time, hardware loop test results are closer to the actual situation.

3) In the training process of neural network algorithm weight matrix, due to the used neural network training sample model doesn't consider the ESP HIL test presenting vehicle status sensor 
signal acquisition noise and other factors, resulting in a car generating steering extreme state; comparing with PID control algorithm, the yaw rate of neural network control algorithm actual value will have a certain deviation in tracking the nominal value process. However, due to the weight matrix can be adjusted online timely, neural network control algorithm can still meet the requirements for ESP algorithm controlling vehicle stability by taking effective control strategy,.

\section{Acknowledgements}

This work was financially supported by the key subject building project (vehicle engineering) of Jiangxi University of Technology.

\section{References}

1. Salehpour S, Pourasad Y, Taheri S H. Vehicle path tracking by integrated chassis control[J]. Journal of Central South University, 2015, 22(4): 1378-1388.

2. Soudbakhsh D, Eskandarian A. Vehicle lateral and steering control[M]//Handbook of Intelligent Vehicles. Springer London, 2012: 209-232.

3. De Novellis L, Sorniotti A, Gruber P. Design and comparison of the handling performance of different electric vehicle layouts[J]. Proceedings of the Institution of Mechanical Engineers, Part D: Journal of Automobile Engineering, 2014, 228(2): 218-232.

4. Gil J J, Díaz I, Ciáurriz P, et al. New driving control system with haptic feedback: Design and preliminary validation tests[J]. Transportation research part C: emerging technologies, 2013, 33: 22-36.

5. Markkula G, Benderius $\mathrm{O}$, Wahde $\mathrm{M}$. Comparing and validating models of driver steering behaviour in collision avoidance and vehicle stabilisation[J]. Vehicle System Dynamics, 2014, 52(12): 1658-1680.

6. Hudha K, Ahmad F, Jamaluddin H, et al. Pid Controller with Roll Moment Rejection for Pneumatically Actuated Active Roll Control (Arc) Suspension System[M]. INTECH Open Access Publisher, 2011.

7. Rill G. Road vehicle dynamics: fundamentals and modeling[M]. CRC Press, 2011.

8. Holloway M, Nwaoha C. Dictionary of Industrial Terms[M]. John Wiley \& Sons, Inc., 2013.

9. Yang X, Xu P. Road Load Analysis Techniques in Automotive Engineering[J]. Yung-Li Lee, Mark E. Barkey, Hong-Tae Kang, 'Metal Fatigue Analysis Handbook: Practical problem-solving techniques for computer-aided engineering', Chapter1, 2011: 1-60.

10. Berry S A, Rhode M N, Edquist K. Supersonic Retropropulsion Validation Experiment in the NASA Langley Unitary Plan Wind Tunnel[J]. Journal of Spacecraft and Rockets, 2014, 51(3): 664-679. 